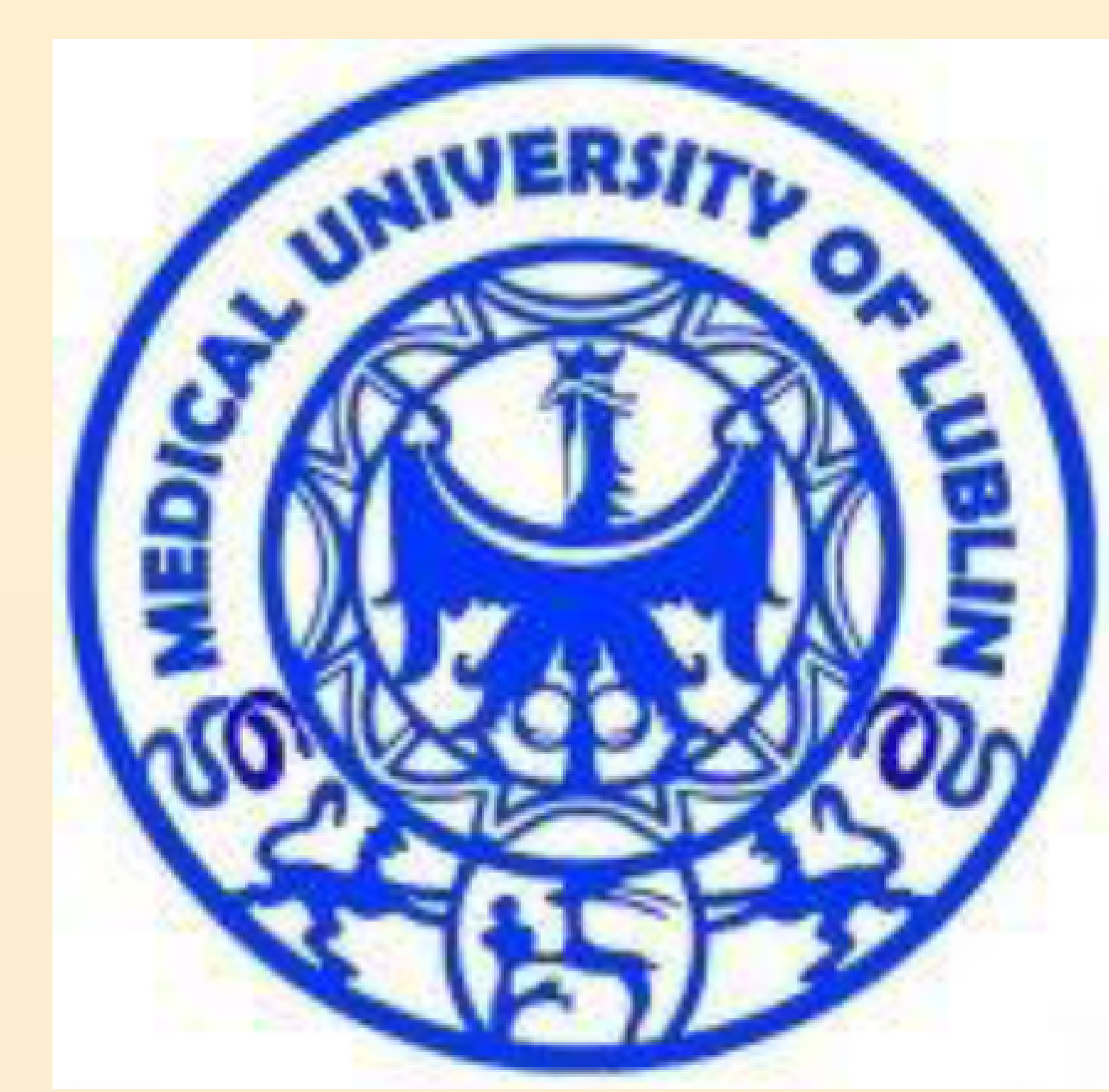

\title{
Accumulation of PD-1-positve T and B lymphocytes in patients with Graves' disease
}

\author{
Aleksandra Pyzik ${ }^{1}$, Ewelina Grywalska², Beata Matyjaszek-Matuszek ${ }^{1}$, \\ Ewa Kiszczak-Bochyńska ${ }^{1}$, Jacek Roliński ${ }^{2}$ \\ ${ }^{1}$ Department of Endocrinology, Medical University, Lublin, Poland \\ 2Department of Clinical Immunology, Medical University, Lublin, Poland
}

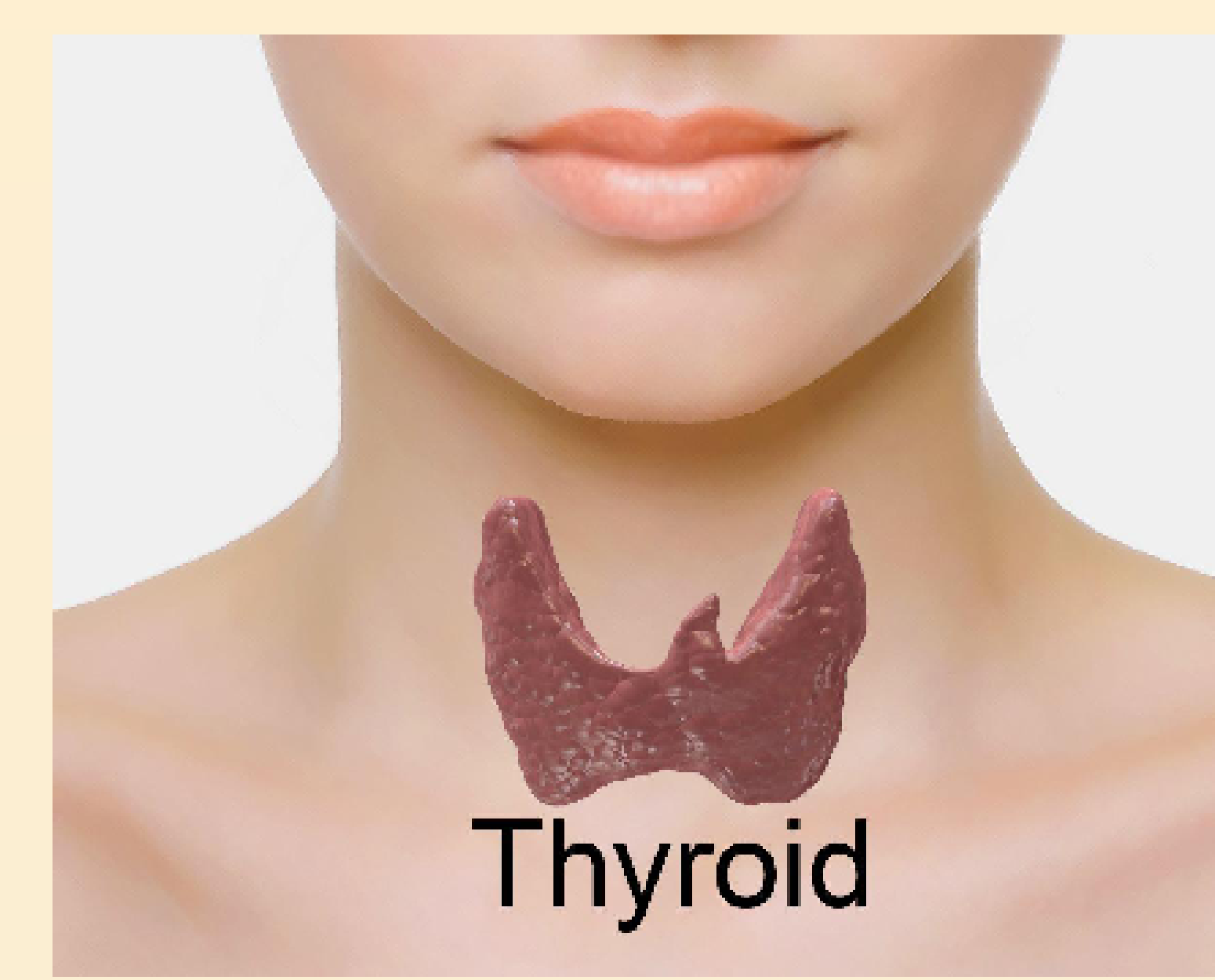

Introduction:

Programmed death-1 (PD-1) is one of the most important inhibitory co-receptors expressed predominantly on activated T and B lymphocytes. Studies show that the PD-1/PD-L1 pathway regulates the induction and maintenance of peripheral tolerance and protects tissues from autoimmune attack in physiological conditions. In addition, it is also involved in various diseases mediated by $T$ cells, such as autoimmunity. Graves' disease is a chronic, autoimmune, organ-specific pathology of thyroid, of not fully known etiology. It is characterized by the presence of vascular goiter and TSH receptor antibodies (TRAbs), sometimes accompanied by extrathyroidal symptoms as orbitopathy or dermopathy or akropathy. Several studies have shown association of PD-1/PD-L1 pathway with several autoimmune diseases: DM1, SLE, RA, psoriasis. To date, no such studies about PD-1/PD-L1 pathway have been performed for Graves' disease (GD).

Fig.1 Expression of PD-1 on CD4+ cells in GD patients

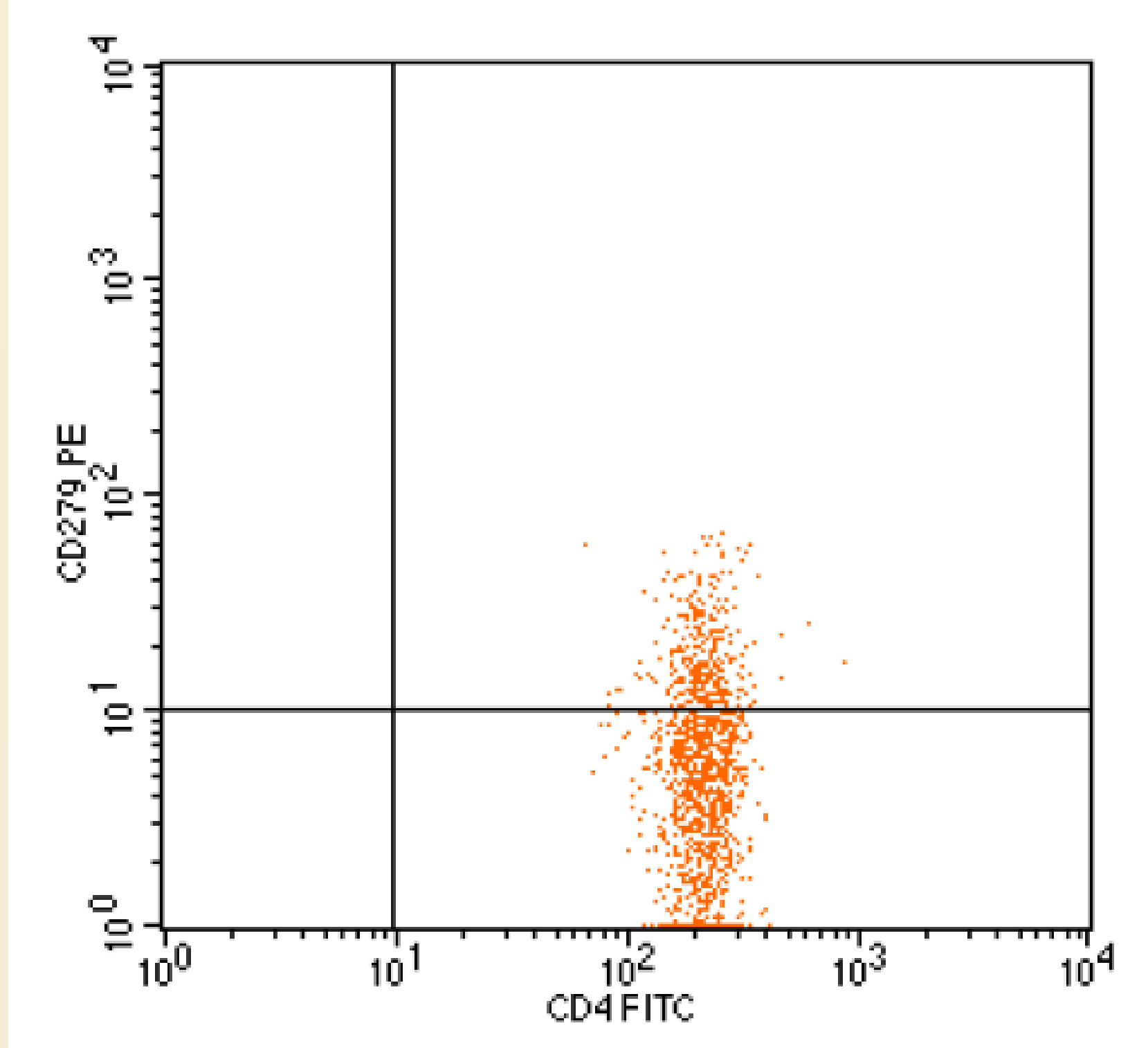

Fig.2 Expression of PD-1 on CD8+ cells in GD patients

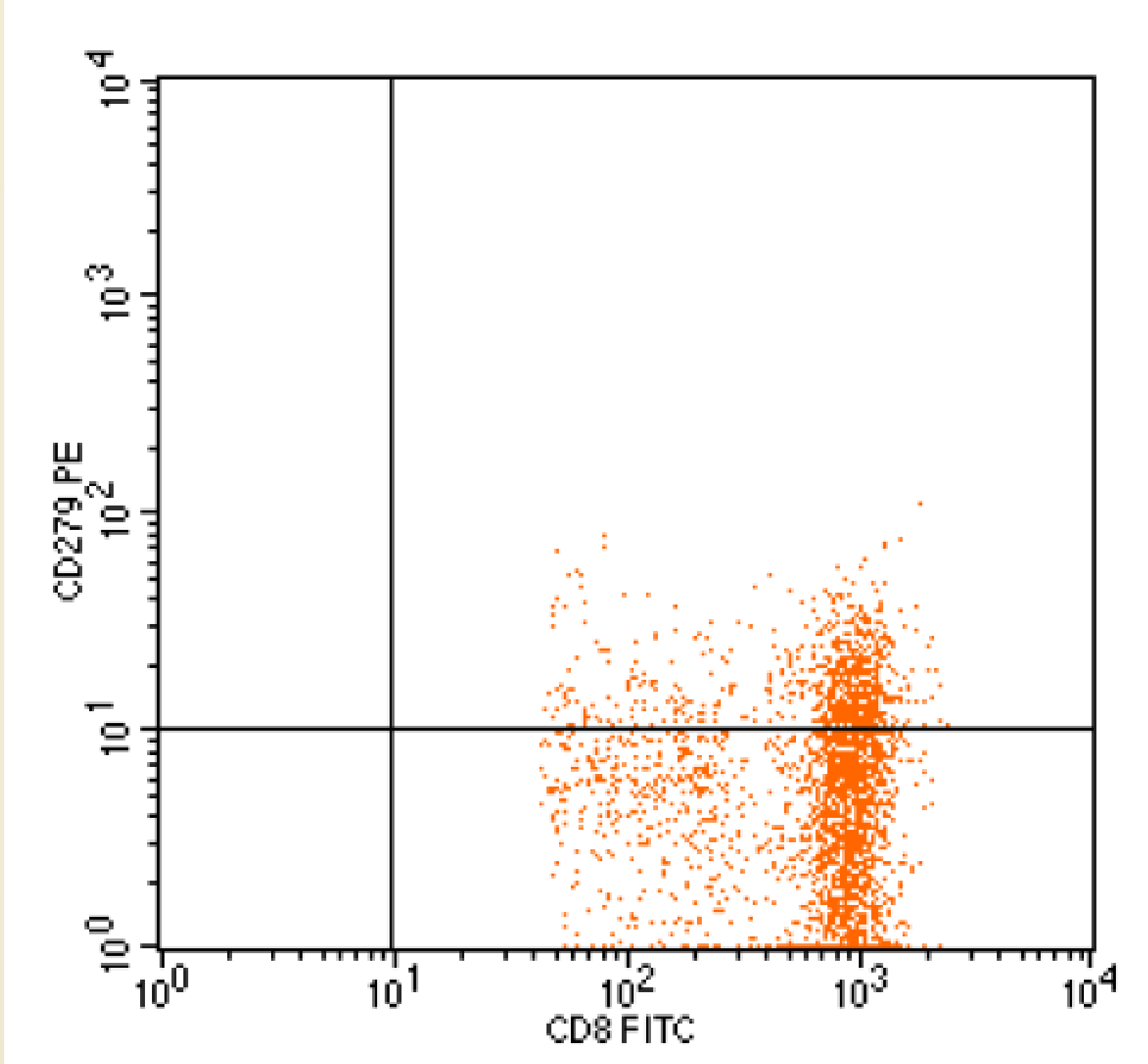

Fig.3 Expression of PD-1 on CD19+ cells in GD patients

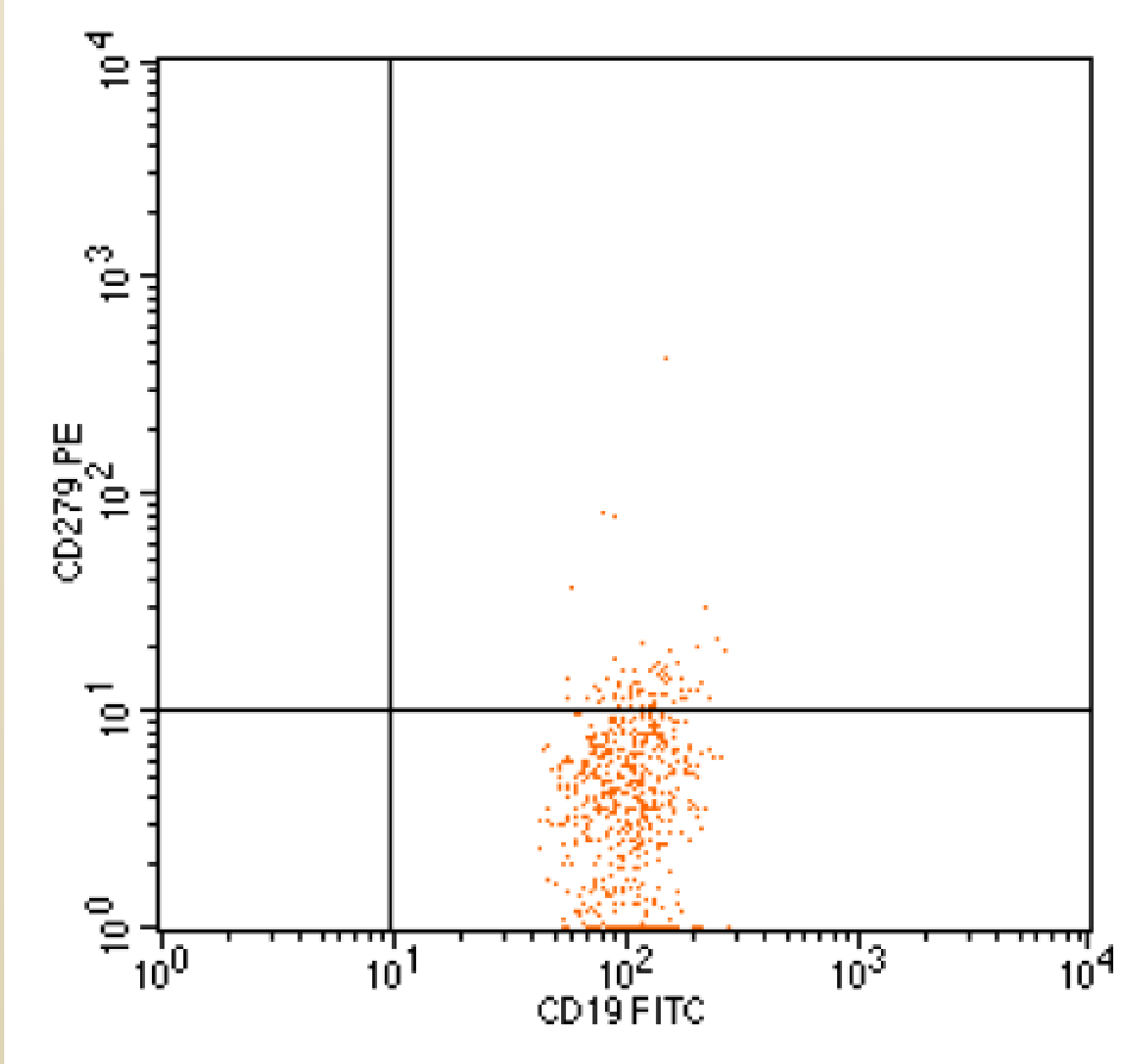

\section{Aims:}

The aim of this research was to describe the frequencies of $T$ and $B$ lymphocytes expressing PD-1 molecules in patients with newly diagnosed hyperthyroidism in the course of GD. The relationships between PD-1+ cells and selected clinical parameters were also assessed.

\section{Material and methods:}

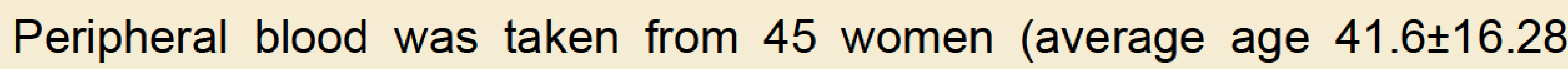
years) with newly diagnosed hyperthyroidism in the course GD at the time of diagnosis of the disease. The control group consisted of 20 healthy volunteers, aged 19-53 years. The expression of PD-1 was analyzed using flow cytometry on $\mathrm{T}$ and $\mathrm{B}$ lymphocytes. The obtained data were statistically analyzed made using STATISTICA ver. 10.0.

\section{Results:}

Among $\mathrm{T}$ cells, the expression of PD-1 on protein level was higher on CD4+ cells in GD patients (mean: $31.54 \pm 13.74 \%$ ) (Fig.1) than in healthy controls (mean: $5.35 \pm 1.54 \%, p<0.001$ ) (Fig.4). Higher frequencies of PD$1+/$ CD8+ cells (Fig.2) and PD-1+/CD19+ cells (Fig.3) in the study group than in healthy volunteers were also observed (mean: $18.71 \pm 10.37 \%$ vs. $3.6 \pm 1.45 \%, \quad p=0.015$ and $12.07 \pm 4.34 \%$ vs. $1.67 \pm 0.84 \%, p=0.017$, respectively) (Fig.5,6). There was a positive correlation of PD-1 expressions on CD19+ $B$ cells $(r=0.43, p=0.026)$ in patients with $G D$, and a positive correlation of the frequencies of $\mathrm{PD}-1+/ \mathrm{CD} 4+$ cells and the serum concentration of anti-TPO antibodies $(r=0.61, p=0.014)$. Interestingly, there was also a positive correlation between the frequencies of PD-1+/CD19+ cells and the serum concentration of anti-TR antibodies $(r=0.53, p=0.019)$. Summary results cytometry in the control group and the study are shown in Table 1.

Tab. 1 Summary results cytometry in the control group and the study

\begin{tabular}{|c|c|c|c|}
\hline parameter & study group $=45$ & control group=20 & $p\left({ }^{*} p<0,05\right)$ \\
\hline $\mathrm{T} \mathrm{CD}^{+} / \mathrm{CD}^{+}[\%]$ & $53.03 \pm 7.55^{*}$ & $44.46 \pm 2.50^{*}$ & 0.00 \\
\hline $\mathrm{T} \mathrm{CD3}^{+} / \mathrm{CD}^{+}[\%]$ & $26.90 \pm 3.69 *$ & $34.36 \pm 3.29 *$ & 0.00 \\
\hline MFI PD-1 on T CD4 ${ }^{+}$ & $163.32 \pm 60.63^{*}$ & $34.33 \pm 18.01^{*}$ & 0.00 \\
\hline $\mathrm{T} \mathrm{CD}^{+} / \mathrm{PD}-1^{+}\left[10^{3} / \mathrm{mm}^{3}\right]$ & $0.34 \pm 0.13^{*}$ & $0.044 \pm 0.017^{*}$ & 0.00 \\
\hline MFI PD-1 on T CD8 ${ }^{+}$ & $414.48 \pm 34.63^{*}$ & $22.85 \pm 13.11^{*}$ & 0.00 \\
\hline $\mathrm{T} \mathrm{CD}^{+} / \mathrm{PD}-1^{+}\left[10^{3} / \mathrm{mm}^{3}\right]$ & $0.11 \pm 0.06^{*}$ & $0.023 \pm 0.011^{*}$ & 0.00 \\
\hline MFI PD-1 on B CD19+ & $139.06 \pm 33.08^{*}$ & $41.62 \pm 17.07^{*}$ & 0.00 \\
\hline $\mathrm{CD} 19^{+} / \mathrm{PD}-1^{+}\left[10^{3} / \mathrm{mm}^{3}\right]$ & $0.03 \pm 0.012 *$ & $0.003 \pm 0.002 *$ & 0.00 \\
\hline
\end{tabular}

Fig.4 Expression of PD-1 on CD4+ cells in GD patients and healthy controls

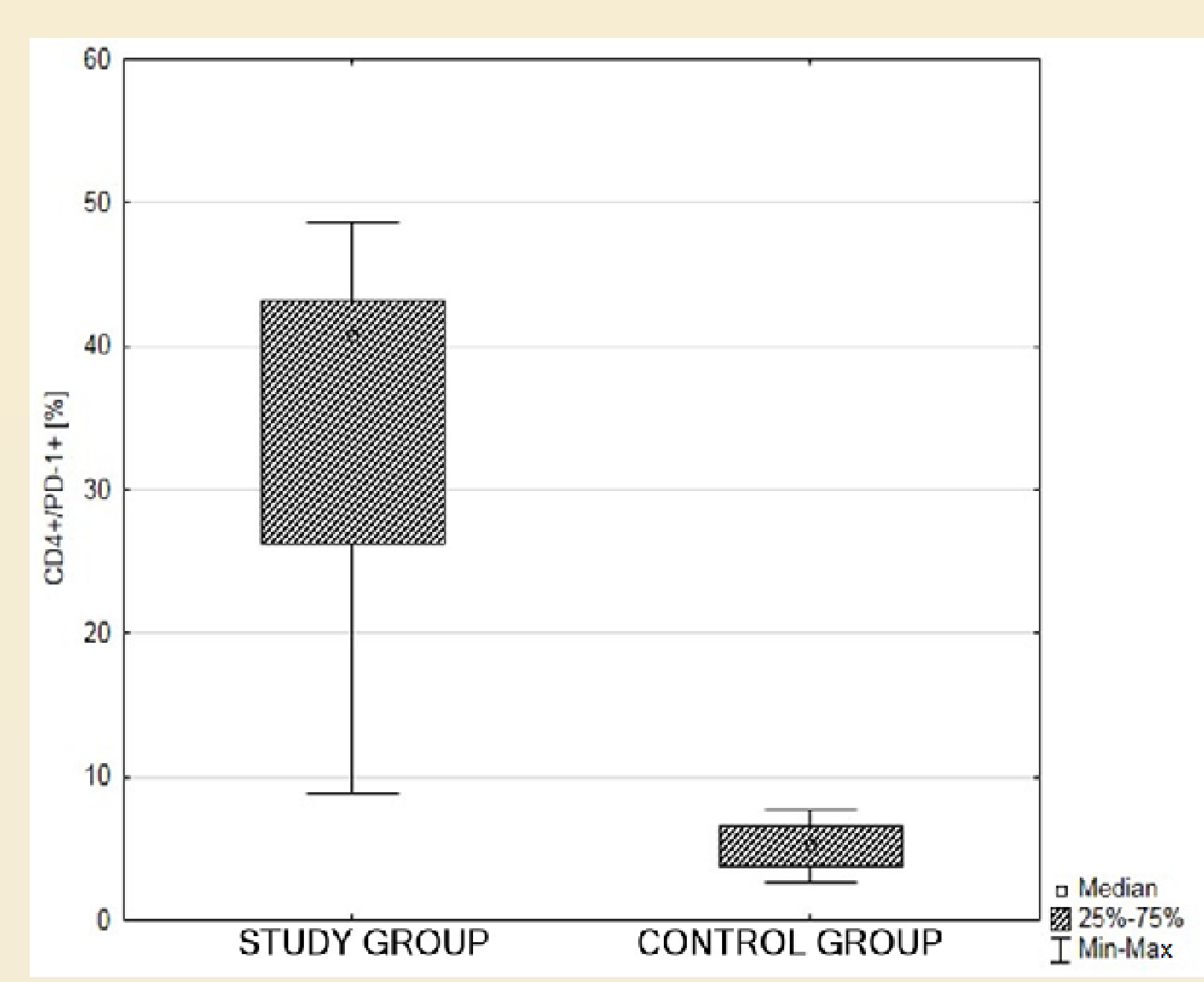

Fig. 5 Expression of PD- 1 on CD8+ cells in GD patients and healthy controls

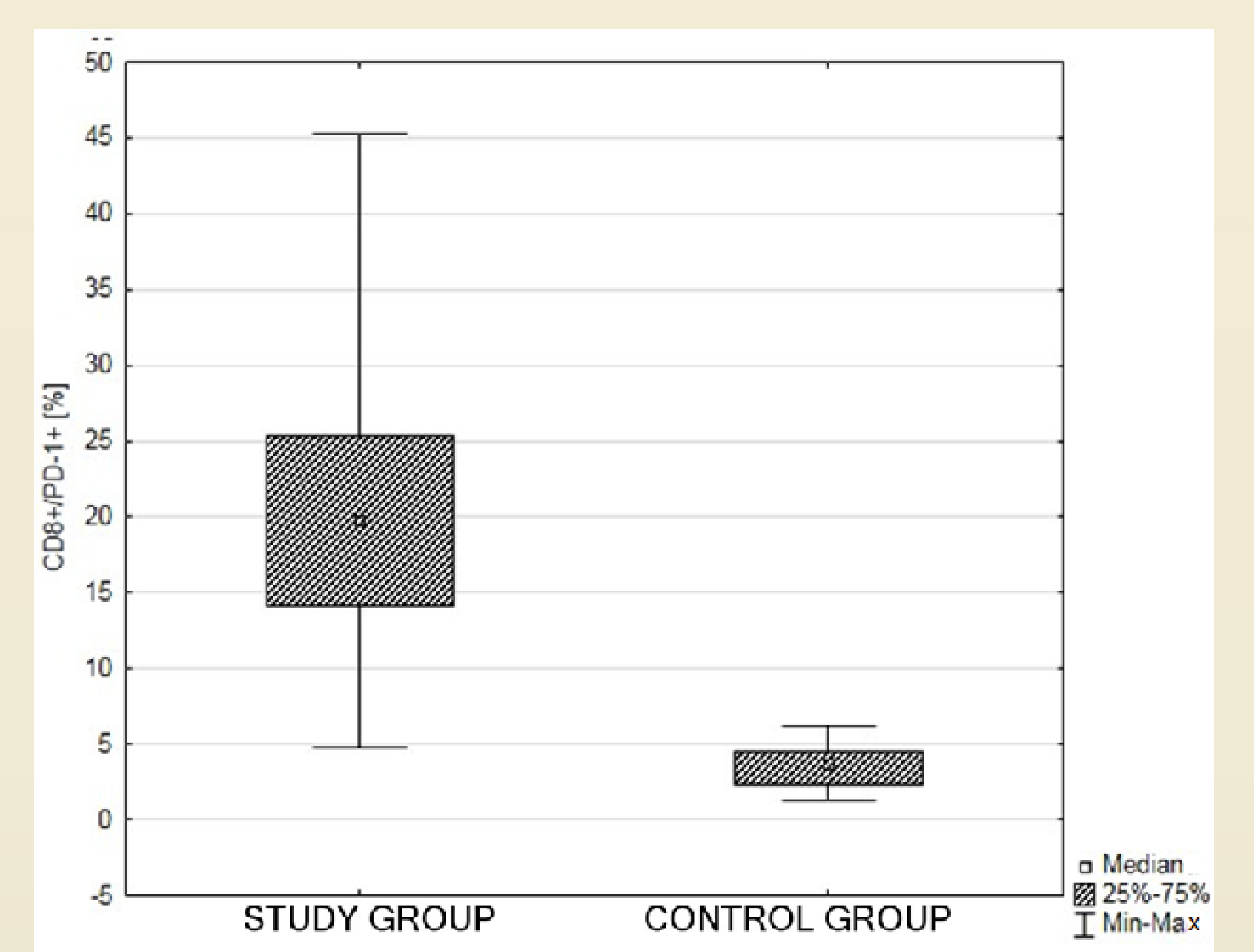

Fig.6 Expression of PD-1 on CD19+ cells in GD patients and healthy controls

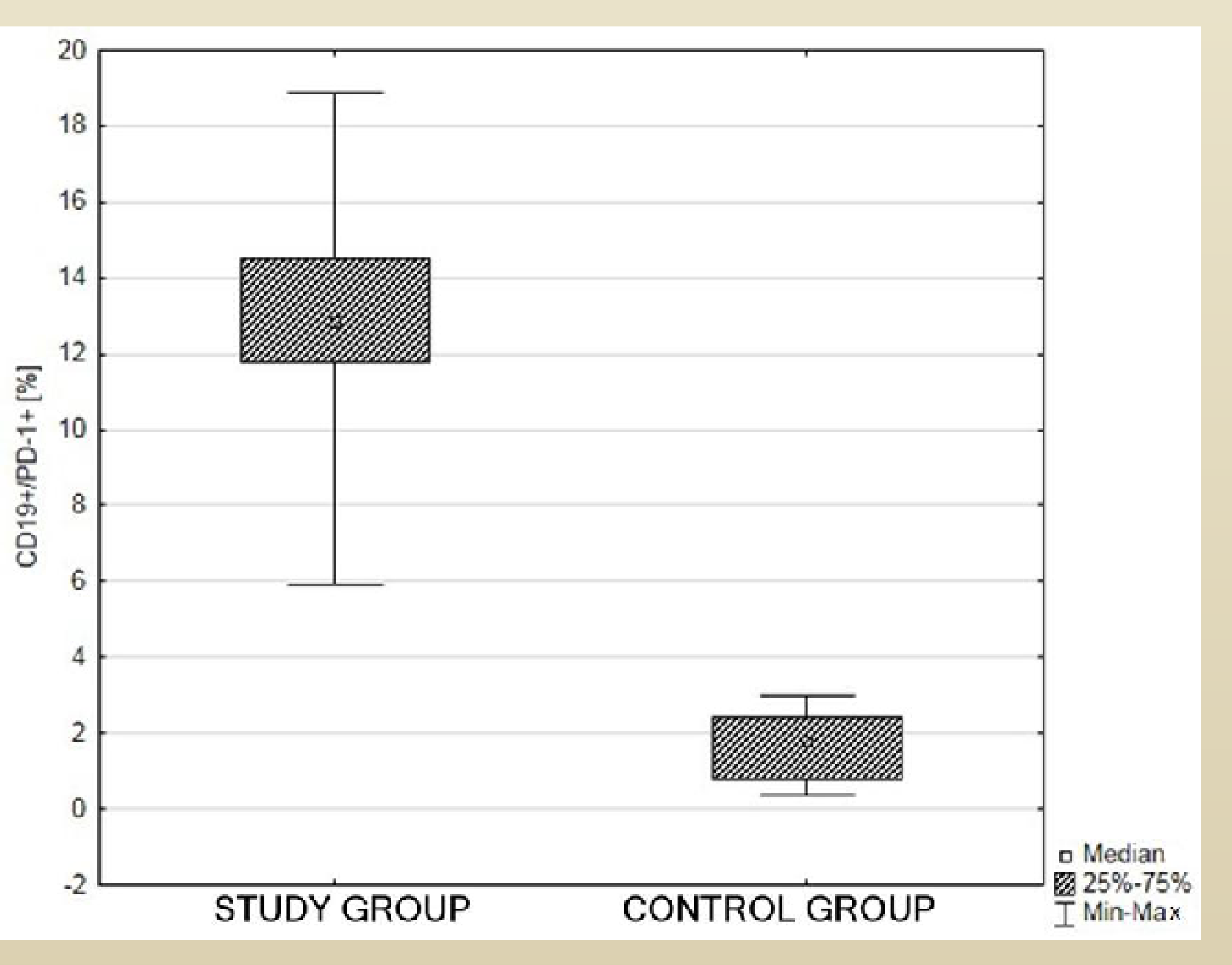

Conclusions:

$>$ We observed high expression of PD-1 on analyzed lymphocytes subpopulations of GD patients compared to healthy volunteers,

$>$ High expression of PD-1 on T and B cells could represent hallmark of immune system reaction to chronic autoantigenic exposition in patients with GD,

$>\mathrm{T}$ - and B-cell exhaustion might play a central role in determining the outcome in autoimmune disease and targeted manipulation of this process could lead to new therapeutic opportunities.

18th European Congress of Endocrinology, Munich, Germany 